\title{
New RPL Protocol for IoT Applications
}

\author{
Nariman Najeeb Hadaya, and Salah Abdulghani Alabady
}

\begin{abstract}
The Objective Function (OF) can be used by the Routing protocol for low power lossy networks (RPL) to construct a Destination Oriented Directed Acyclic Graph (DODAG) based on routing metrics. The standard OFs suffer from long hops when selecting the route, which may cause consume the node's energy faster. In this paper, we suggest an improvement of RPL OF that considers three metrics. The results show that the proposed protocol increases network lifetime by reducing energy consumption, increasing efficiency, increasing Packet Delivery Ratio (PDR), and decreasing packet loss ratio. In terms of PDR, packet loss ratio, and average power consumption, the best performance of the proposed protocol is shown in the network with 70 nodes and when the transmission range is $50 \mathrm{~m}$. Compared with the MRHOF, the proposed protocol increased the PDR by $58.425 \%$, decreased the packet loss ratio by 0.21765 , and decreased the total power consumption by $181.815 \mathrm{~mW}$. In terms of the average Expected Transmission Count (ETX) the best performance of the proposed protocol is shown in the network with 60 nodes and the transmission range is $40 \mathrm{~m}$. The proposed protocol reduced the average ETX by 49 compared to the MRHOF.
\end{abstract}

Index Terms-RPL, Low-power and Lossy Networks, Objective Function (OF), MRHOF, IoT, Routing Protocol.

\section{INTRODUCTION}

$\mathbf{T}$ HE RPL is Low Power Lossy Networks (LLNs networks) routing protocol was created to handle resourceconstrained equipment, routing in smart cities, manufacturing, smart homes, and many applications [1]. When the resources in these devices are limited, poor quality paths can quickly deplete the limited resources. The routing protocols like Open Shortest Path First (OSPF), Ad Hoc on Demand Vector(AODV), Optimized Link State Routing(OLSR), and Dynamic Source Routing (DSR) can't overcome the LLNS networking's constraints like low data lost, low data rates, low power consumption, and network stability [2].

The RPL protocol is built on the IEEE 802.15.4 MAC layer and the physical layer. There are two types of nodes in the RPL network. The sink node, gathers data from all network nodes. The source nodes, on the other hand, collect data from various sensors. After the RPL node routing topology is created, all the common nodes in the network will periodically send their data to the sink node. A Destination Centered Directed Acyclic Graph (DODAG) is the basis of the creation of RPL networks. The standard Objective Function (OF), on the other hand, has

Manuscript received May 6, 2021; revised October 15, 2021. Date of publication March 1, 2022. Date of current version March 1, 2022. The associate editor prof. Toni Mastelić has been coordinating the review of this manuscript and approved it for publication.

Authors are with the Computer Engineering Department, University of Mosul, Iraq (e-mails: nariman.enp97@student.uomosul.edu.iq, eng.salah@uomosul.edu.iq).

Digital Object Identifier (DOI): 10.24138/jcomss-2021-0094 several problems that can be traced back to the use of only one metric such as selecting inefficient routes like paths containing nodes with small residual energy, which may consume the node's energy faster than other nodes. The other problem of RPL is unbalanced chosen by parents that make bottleneck nodes that cause more network delay and high packet loss ratio because of the high congestion nodes, especially the nodes that locate near to the sink node. We attempted to address these issues in this research by proposing an enhanced RPL objective function that takes into account three metrics (Load, Residual Energy, and ETX metrics) rather than only one. The tool used in this paper is the Cooja simulator.

The remainder of this paper is organized as follows. First, in Section II, the DODAG Creation is explained. In section III we explained the problem statement. Section IV reviews the related work that attempts to improve the RPL objective function. Section $\mathrm{V}$ defines the proposed objective function model to enhance the RPL protocol. In section VI, the simulation results are evaluated with three network scenarios $(50,60$, and 70 nodes) and show the results of the proposed protocol and compare it with the RPL MRHOF with different transmission ranges $(40 \mathrm{~m}, 50 \mathrm{~m}$, and $60 \mathrm{~m})$, in terms of the PDR, average packet loss ratio, total power consumption, and average ETX. Finally, section VII concludes the paper.

\section{The DODAG CREATION}

A Destination Oriented Directed Acyclic Graph (DODAG) is the basis of the creation of RPL networks. To build and optimize the path, the OFs choose the best parent nodes. The DODAG relation produces a series of specific edges, as demonstrated in Figure 1. The RPL DODAG is constructed by linking the leaf node to the sink (root) node via a sequence of pathways. The topology's structure lacks a defined loop. Each node in a network has a rank value that reflects its position in comparison to the sink node. The rank value is usually an integer number, and the DODAG sink node holds the minimum rank value for the whole network. The rank value raises the path relation from the root (sink) node to the leaf node downwards, while it decreased from the leaf node to the root node upwards. The root node in the DODAG network collects all of the data. The network's nodes will communicate with not only their own parents, but also their neighbors. The node ranking is the scalar expression within a DODAG of the position of that node. The OF will be in charge of determining the exact rank measurement. During the development of the DODAG network, each router node can choose several potential parent nodes based on its OF. The OF defines how RPL nodes choose and improve routes within an RPL instance [3] [4]. 


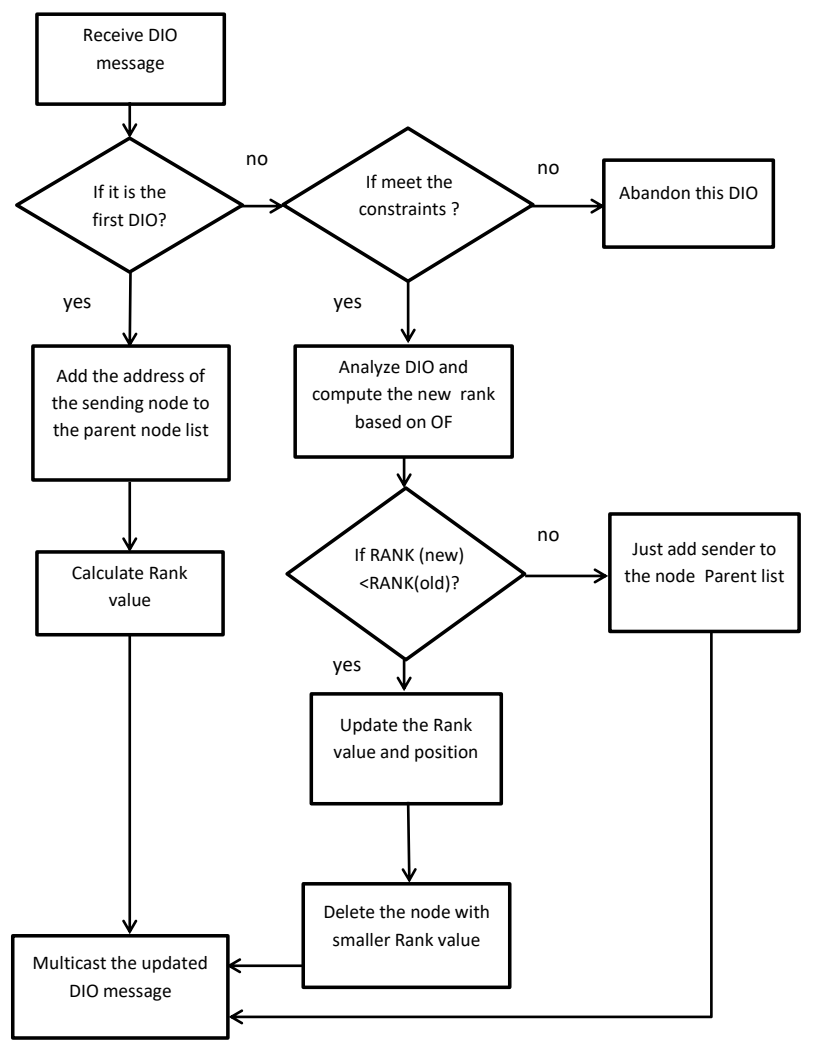

Fig. 1. The process to build DODAG

RPL uses objective function (OF) operations to choose a preferred parent for traffic forwarding. The OF considers a variety of parameters and constraints of nodes and networks to choose the best route. The Internet Engineering Task Force (IETF) Routing Over Low power and Lossy networks (ROLL) working group has two standard OFs, named OF zero (OF0), and Minimum Rank with Hysteresis Objective Function (MRHOF) [5] [6]. In the RPL when constructing the DODAG and select parents, the RPL may suffer from select inefficient routes like paths contain nodes with small residual energy, which may cause consume the nod's energy faster than other nodes. The other issue with RPL is that it is unbalanced by parents, resulting in bottleneck nodes that create increased network delay and a high packet loss ratio due to high congestion nodes, particularly those located near the sink node. To improve network performance, many articles have been presented to improve RPL efficiency.

\section{The Problem Statement}

In RPL, the OFs that are used to select the forwarding node (parent node), may suffer from the problem of long hops, when the network becomes heavy because of the large number of nodes when the size of the network increases. This is due to the single metric RPL objective function, such as hop count, or ETX. In addition, a flocking effect can be a result of the parent selection scheme in RPL, which refers to the incidence of attracting nodes and continuous switching from one parent to another. As a consequence, this flocking phenomenon would have a major effect on QoS provisioning

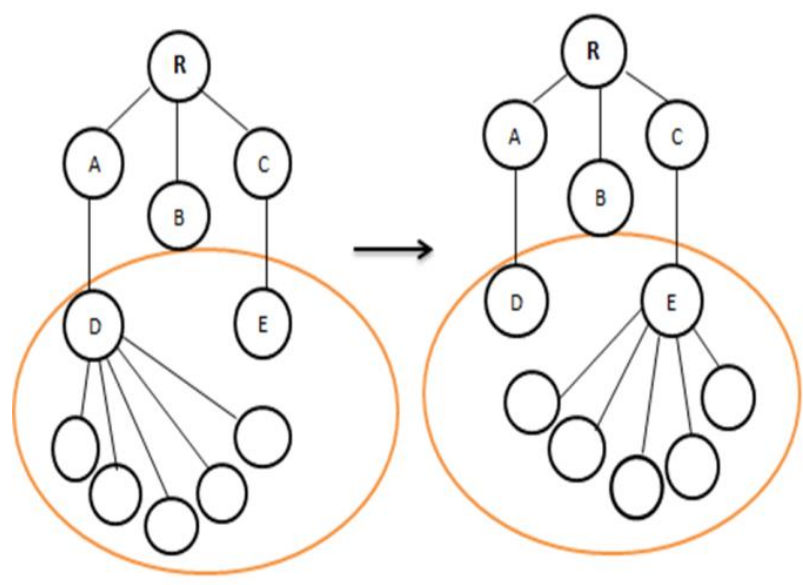

Fig. 2. The Flocking Phenomena

that essentially restricts the services of the IoT application. Since selecting parents in the OF0 does not consider link quality and only tries to find a path with minimum hop count, this metric is not efficient enough because it does not take into consideration the long hop that negatively affects energy consumption and QoS. While MRHOF only takes the ETX metric without consideration of other metrics, this harms the network performance. According to the RPL MRHOF objective function, each node chooses their preferred parent based on the lower rank which is based on a lower ETX value. This parent selection process may result in the flocking effect, which attracts nodes quickly and disrupts network balancing [6].

Consider the network in Figure 2 to have a better understanding of the situation. Nodes D and E, which have rank values of 10 and 11 , respectively, are candidate parents of multiple nodes in this topology. The shared transmission range area of the nodes is shown in the orange circle. As the preferred parent, since node $\mathrm{D}$ has a lower rank than node $\mathrm{E}$, all the relevant nodes in their area will choose $\mathrm{D}$ as their preferred parent because it broadcasts the lowest route cost. After a time, when the parent $\mathrm{E}$ broadcasts a lower rank than parent D and all of the nodes in the coverage area satisfy the rank policy, all of the relevant nodes switch to node $\mathrm{E}$ as their preferred parent. Nodes will switch their preferred parent again if a new node enters the network and broadcasts the better rank value. As a result of this scenario, flocking occurs, with repeated parents quickly moving (switching) from one to the other parent. In large-scale IoT networks, the flocking effect causes frequent parent changes, which leads to network instability. Consequently, the network will not run smoothly, and it will have a substantial influence on QoS needs.

RPL has many strong specifications, including a quick initialization, self-healing, and loop-free topology. The load imbalance, on the other hand, is regarded as a major weakness in RPL protocol. In addition to universal delivery, RPL specifically defines a non-uniform implementation of largescale LLNs, resulting in unequal data traffic. As a result, the energy of overburdened nodes will deplete even more quickly 
than other nodes in the network. However, if the overloaded node is a bottleneck node (the nodes that lie near the root, the first hop), the problem is even more serious. The nodes near the sink node suffer from depleted energy faster than other nodes, because the MRHOF protocol depends on the ETX only to build DODAG and select the parent. We proposed the Load metric as a solution for the problem of the bottleneck nodes because it makes a load balance that extends the network lifetime, while the RE metric is a solution for both flocking phenomena and bottleneck problems.

\section{RELATED WORK}

The authors in [7] attempted to optimize the lifetime of the network, by choosing the next hops to the DODAG sink node according to the residual energy of the node. Nodes are added time period spend in each state from node energy (idle, receive, sense, transmit, compute). The cost of the calculated path would then equate to the residual energy of the path's node. Nodes would then pick a neighbor which path to the sink node holds the best maximum value of a lifetime and according to the new proposed OF. The major downside of this approach is an observed deterioration of the efficiency of the transmission inefficient routes with poor radio links that may be used.

The authors in [8] proposed a new approach for increasing network reliability while maintaining a balanced energy usage across network nodes. The authors created the Estimated Lifetime (ELT) metric to determine how long a node will last before it runs out of energy. The proposed OF ensured that topology nodes had balanced power consumption independent of their distances from the DODAG sink, based on simulation performance. However, there is an extra delay.

In [9], the authors mentioned their suggested Bounded Degree RPL solution (BD-RPL). Instead of using the children count as a measure, they used it to set a limit for DODAG by limiting the children count a favored parent can have. The research scheme's strength is that it has no additional overhead when compared to RPL. It is also unaffected by the radio link quality metric. According to the researchers, BD-RPL improved PDR by $10 \%$, reduced power consumption by $50 \%$, and reduced delay by $60 \%$ when compared to standard RPL.

In [10], the paper proposes a new RPL metric based on the approximate node Queue Backlog to increase overall throughput efficiency, maintaining regular delay, and being compatible with multiple network equipment. In [11], the authors proposed a new OF called (LB-OF), which attempts to balance the distribution of loads across all nodes in LLNs, particularly bottleneck nodes. The authors achieved this by substituting the ETX metric, which took into account the children count in the rank calculation and thus favored parent selection. The proposed technique allowed the children count for overstretched nodes to be balanced in relation to MRHOF, ensuring node lifetime optimization. Despite this, the authors did not investigate the proposed OF effect on the PDR and latency.

The authors in [12] proposed an OF dependent on a composite metric using the OF-EC fuzzy logic framework. As input fuzzy method, they chose HC, ETX, and power metrics, but computed power consumption differently. Therefore, both transmitter and receiver consumed energy, processing energy, and both maximum and low energy usage modes were used to assess the energy consumption. The authors say that, with comparison to other RPLs, the proposed OF-EC efficient performance in calculating PDR, delay, the lifetime of the network, energy consumption, and convergence time. The authors in [13] proposed the Composite Metric OF (CMOF), which mixes latency and ETX route metrics that are supposed to increase both latency and PDR by choosing better communication and fewer traffic routes. In reality, while ETX will guarantee the use of secure connections, by summing the time packets spend in the transmitted queue and the time is taken to reach the link, latency will quantify both traffic and contention at any node. The paper adjusts transmission power to assist establish the network topology and reducing congestion to improve latency and PDR, but in the simulation, they test only the network with 12 nodes, many network scales should be taken to accurately determine the protocol efficiency.

The authors in [14], presented an objective function called (OFER) depending on a hybrid routing metric to get an optimal parent choice. A collection of weighted metrics, including packet loss, connection consistency ETX due to queue occupancy, the node's remaining duration to live, and latency, are combined with the CER metric. In comparison to standard RPL, EN-RPL reduces packet loss by up to 37 percent and reduces power consumption by up to 46.5 percent, according to simulation data. In terms of network stability, the evaluation findings of OF-ER exceed state-of-the-art load balancing based OF. The paper argues that not only could OF-ER minimize queue loss and power consumption, but also extend the planned lifetime of the node. But, the authors failed to mention the metric impact on traffic or its ability to support different network sizes.

\section{The Proposed Objective Function}

In RPL, the OFs that used to select the forwarding node (parent) may suffer from the problem of long hops, when the network becomes heavy because of the large number of nodes when the size of the network increases [15], this due to the single metric RPL objective function, such as hop count or Expected Transmission Count (ETX). In addition, a flocking effect can be a result of the parent selection scheme in RPL, which referred to the incidence of attracting nodes and continuously switching from one parent to another, as a consequence, this flocking phenomenon would have a major effect on QoS provisioning, which essentially restricts the services of the IoT application. Since selecting parents in the objective function zero (OF0), does not take link quality into consideration and only tries to find a path with minimum hop count, this metric is not efficient enough because it does not take into consideration the long hop that is effect negatively in energy consumption and QoS, while MRHOF is only taking the ETX metric without consideration of other metrics, this affects negatively on the network performance. Since the objective function, routing parameters, constraints, 
and local policies to be used in the parent selection and route establishment process can be freely chosen in the RPL, in order to provide high flexibility, therefore, we designed a new objective function that combines three metrics: node residual energy, load metric, and ETX, the residual energy metric solve the problem of the selecting the nodes with low energy, so it prevents the network drop early. The load metric prevents selecting the bottleneck nodes therefore the proposed protocol solved the problem of the flocking phenomena and the bottleneck problem. Every node when it selects parent and constructs the DODAG from the candidate parent nodes, should select the node that has minimum ETX, minimum load metric, and maximum node residual energy.

\section{A. Calculate Residual Energy}

As a representation of the network lifetime, we used the residual energy metric (RE). Therefore, when construct DODAG and select a parent every node should not select a parent that has low residual energy to prevent choosing nodes that have low energy. Based on its various operating modes, the energy consumption of each node is calculated. These modes are usually listening mode which includes (listen +receive $(\mathrm{RX})+$ idle modes), transmission mode (TX mode), processing mode (CPU mode), and low power mode (sleep mode). The current energy consumption can be calculated as Eq.(1) [3].

$$
\begin{array}{r}
E C(x)=P_{\text {sleep }}(x) \times T_{\text {sleep }}(x)+P_{t x}(x) \times T_{t x}(x)+ \\
P_{\text {cpu }}(x) \times T_{\text {cpu }}(x)+P_{\text {les }}(x) \times T_{\text {les }}(x)
\end{array}
$$

where EC(x) is the energy consumed by node (x). $P_{\text {mode }}$ is the power consumption modes $\left(P_{l e s}, P_{T x}, P_{c p u}, P_{\text {sleep }}\right)$. $T_{\text {mode }}$ is the duration of time in each mode $\left(T_{l e s}, T_{T x}, T_{c p u}, T_{\text {sleep }}\right)$. Equation 2 can be used to calculate residual energy.

$$
R E(x)=\operatorname{EMax}(x)-E C(x)
$$

where $\operatorname{RE}(\mathrm{x})$ is the residual energy of the node $(\mathrm{x}), \operatorname{EMax}(\mathrm{x})$ is the maximum energy of the node $(\mathrm{x})$.

\section{B. Calculate Load Metric}

The network data traffic is a quantity of data transmission at a specific amount of time across the network. A load balancing method was proposed by the authors in [11]. The load metric can be used to make balance of the data traffic in the network. Depending on the number of children present throughout the parent node, the load is calculated. The DODAG nodes broadcast all participant nodes with DIO message. The participant node or sender node computes for each preferred parent the number of children. Finally, based on the cumulative children number present in the link, the DODAG generate the rank. The participant node selects the parent from the candidate parent list, based on the load metric. The load metric can be calculated as Eqs. (3) and (4).

$$
N T=\sum_{i=1}^{n} C N(i)
$$

where $\mathrm{N}$ is the node traffic, $\mathrm{CN}$ is the children number.

$$
L(P x)=\sum_{N=1}^{n} N T(N)
$$

where $\mathrm{L}(\mathrm{Px})$ is the load on parent $(\mathrm{x})$.

The number of children that connect with this node is used to determine node traffic.

\section{Calculate the Proposed Objective Function}

The Objective Function (OF) determines how to construct the DODAG and selects the parents, in the RPL network to optimize the path. The proposed objective function composite three metrics to increase energy efficiency. This objective function focuses on issues like data traffic in multi-point to point communications. The bottle-neck occurs nearby sink node. Becoming a chosen parent, for more children means unbalanced load, high congestion, more loss packet, and more overhead, thereby wasting its own energy even faster than other preferred parents did. A load metric has been proposed to solve this problem, providing each chosen parent with how many children they have. On the basis of that, in the rank measurement, we take into consideration the number of children. Every node when it selects parent and construct the DODAG from the candidate parent nodes, should select the node that has minimum ETX, minimum load metric, and maximum node residual energy $(\max \mathrm{RE}+\min \mathrm{ETX}+\min$ Load).

\section{Network SETUP AND Simulation Result ANALYSIS}

In this paper, the Cooja /Contiki3 simulator has been used for the simulations. In the Contiki area, this simulator is integrated and can run the very same executable as those for real-life nodes. The 'Unit Disk Graph Model (UDGM)' was used. The smart home is the case study of our proposed, the transmission range (maximum distance where a packet can still be received) is taken $(40 \mathrm{~m}, 50 \mathrm{~m}$, and $60 \mathrm{~m})$, and interference distance is designed for this model (the maximal distance of the collision domain) is $100 \mathrm{~m}$. For the MAC protocol, the CSMA protocol was used. The simulation area is $100 \mathrm{~m} \times$ $100 \mathrm{~m}$, the type of the nodes is sky motes, with three network topologies (50, 60 and 70 nodes). In each network we used three transmission ranges $(40 \mathrm{~m}, 50 \mathrm{~m}$, and $60 \mathrm{~m})$, with random distribution of nodes. Table 1 shows the network parameters that used in this simulation. The time of simulation for each network is $1200 \mathrm{sec}$. We applied the MRHOF OF and the proposed protocol on each network and several metrics are calculated such as, Total Power Consumption, Packet Delivery Ratio (PDR), Packet Loss Ratio, and ETX.

\section{A. Packet Delivery Ratio}

PDR is "defined as the number of data packets received by the sink (root) node to the number of data packets sent to the sink (root) node". Figure 3 shows the packet delivery ratio (PDR) with the number of nodes (50, 60, 70 nodes), each node implemented with various transmission ranges $(40 \mathrm{~m}, 50 \mathrm{~m}$, and 
TABLE I

Simulation Network PARAMETERS

\begin{tabular}{c|c}
\hline Parameter & Value \\
\hline Operating System & Ubuntu 14.04, Instant Contiki 3 \\
\hline Simulator & Cooja simulator \\
\hline Simulation area & 100m $\times 100 \mathrm{~m}$ \\
\hline Transmission range & $100 \mathrm{~m}$ \\
\hline Interference range & IPv6 \\
\hline Radio model & Unit Disk Graph Medium (UDGM) \\
\hline Packet format & 50, 70 nodes \\
\hline Number of nodes & 127-byte payload \\
\hline Type of nodes & MSP430 \\
\hline Packet size & UDP/IPv6 \\
\hline microcontroller & Enabled \\
\hline Transport layer protocol & Tmote (Sky mote) \\
\hline MAC layer reliability & CSMA/CA \\
\hline Node type & Standard IEEE 802.15 .4 MAC \\
\hline Data Link layer & Standard IEEE 802.15 .4 PHY \\
\hline MAC &
\end{tabular}

60m), each node is sending 20 packets in each MRHOF and the proposed protocol.

The network with 50 nodes in the first topology, as seen in Figure (3a), has a transmission range of $40 \mathrm{~m}$. The packet delivery ratio in the MRHOF is around 74.695 , while it is 96.02 in the proposed protocol. The packet delivery ratio in the MRHOF is around 90.1, and in the suggested protocol is 97.145 while the transmission rage of $50 \mathrm{~m}$. The packet delivery ratio in the MRHOF is around 93.88 while the transmission rage of $60 \mathrm{~m}$, but it is 97.55 in the proposed protocol. The second topology, as seen in Figure (3b), has 60 nodes and a transmission range of $40 \mathrm{~m}$. The packet delivery ratio in the MRHOF is approximately 34.31, although it is 83.475 in the proposed protocol. The packet delivery ratio in the MRHOF is around 53.985 for a transmission range of $50 \mathrm{~m}$, and 94.575 in the proposed protocol. The packet delivery ratio in the MRHOF is around 83.985 for a transmission range of $60 \mathrm{~m}$, and 96.525 in the proposed protocol. The network with 70 nodes, with a transmission range of $40 \mathrm{~m}$, is seen in the third topology, as seen in Figure (3c). The results show that in the MRHOF, the packet delivery ratio is about 31.2, while in the suggested protocol, it is 85.505 . The packet delivery ratio in the MRHOF is around 34.69 for a transmission range of $50 \mathrm{~m}$, and it is 93.115 in the proposed protocol. In case the transmission range is $60 \mathrm{~m}$, we found that the packet delivery ratio in the MRHOF is about 94.35 , and in the proposed protocol is 97.1. Figures 3a, 3b, 3c show that the efficiency of the proposed protocol increased when the number of nodes increased especially with the low transmission ranges $(40,50)$. In the standard protocol and proposed protocol, the PDR increased when the transmission range increased because the network hop count to the sink node decreased. In the case of the transmission range is $60 \mathrm{~m}$, most of the nodes reach the sink in one hop, and the rest of the nodes need two hops, so the effect of the proposed protocol is not visible.

\section{B. Total Lost Packet}

Figure $4(\mathrm{a}, \mathrm{b}, \mathrm{c})$ shows the total lost packet for three network topologies $(50,60$, and 70 nodes), each network implemented with various transmission ranges $(40 \mathrm{~m}, 50 \mathrm{~m}$, and

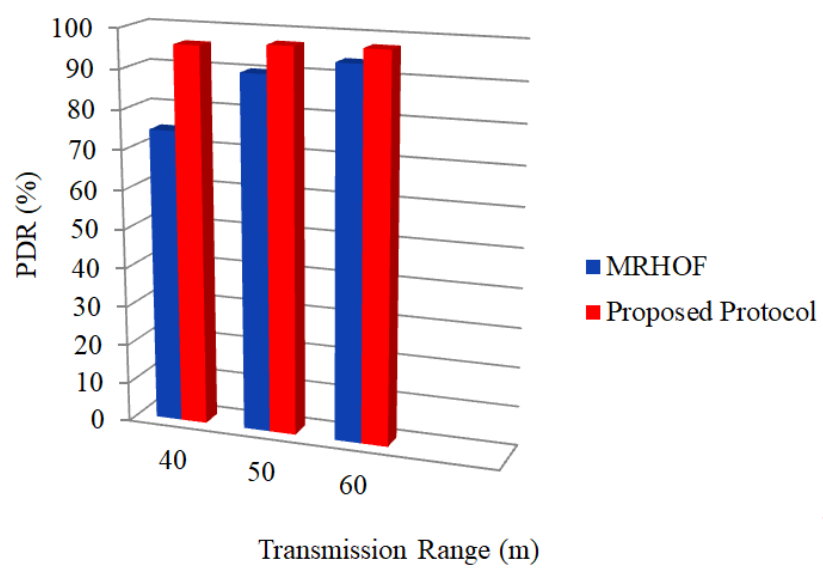

(a) 50 node

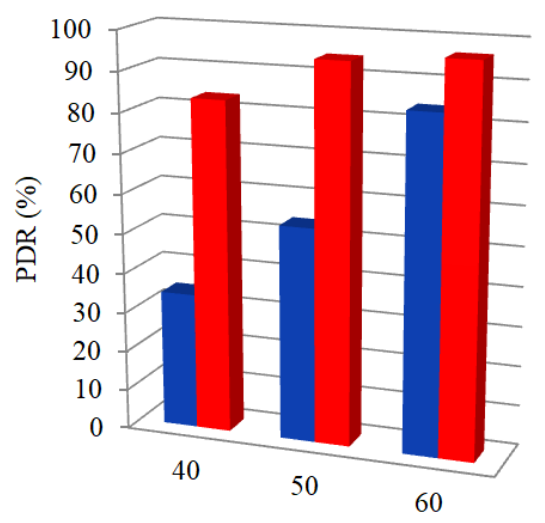

- MRHOF

- Proposed Protocol

Transmission Range (m)

(b) 60 node

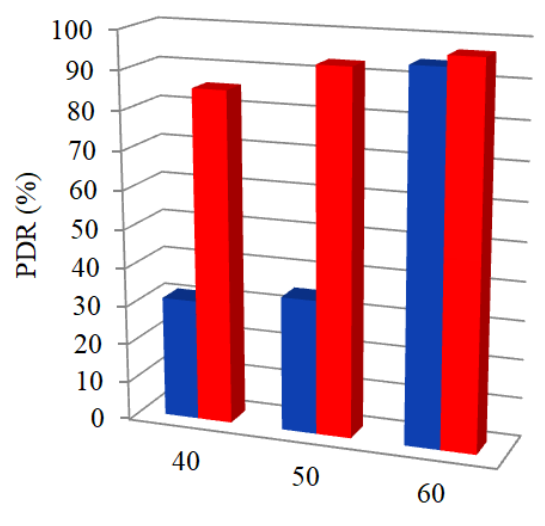

- MRHOF

- Proposed Protocol

Transmission Range (m)

(c) 70 node

Fig. 3. (a, b, c) The packet delivery ratio with various transmission ranges for three network topologies $(50,60,70$ nodes $)$.

$60 \mathrm{~m}$ ), and each node is sends 20 packets for each MRHOF and a newly proposed protocol. When the PDR increased the total lost packet decreases it can reach zero in some cases(transmission range of $60 \mathrm{~m}$ ) because the network has a good efficiency with the fewer hop count routes. 


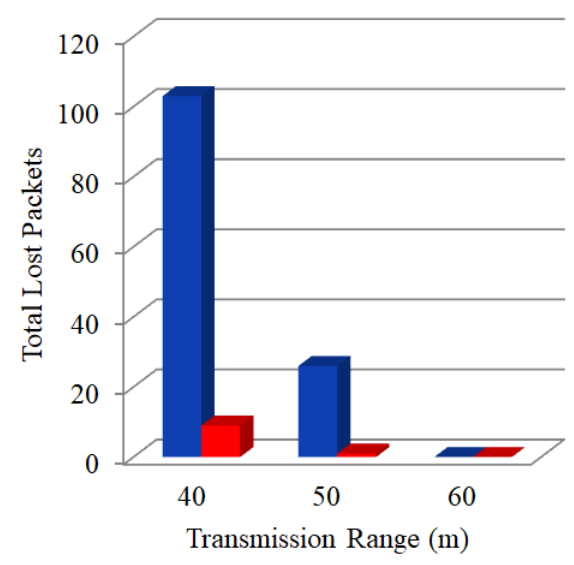

(a) 50 node

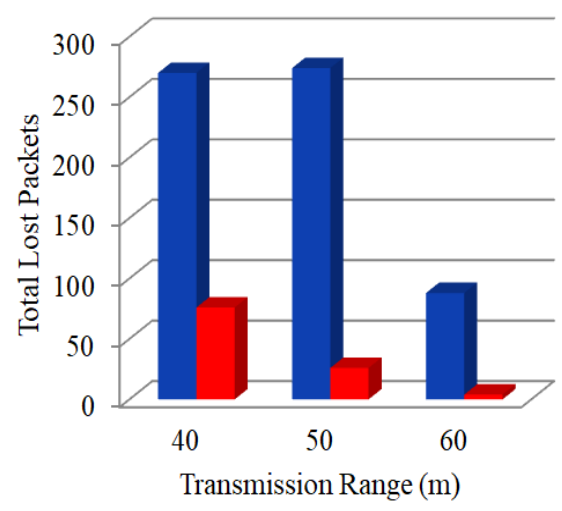

(b) 60 node

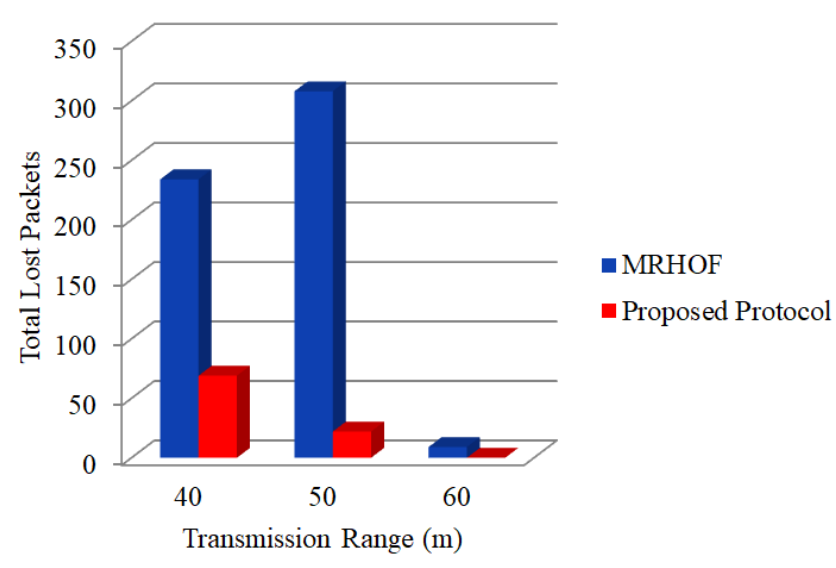

(c) 70 node

Fig. 4. (a, b, c) The total lost packet with various transmission range for three topologies $(50,60,70$ nodes $)$

\section{Total Power Consumption}

Because the sensor nodes are battery-powered, the total consumption is an important parameter for an efficient sensor network operation, therefore we measured this parameter in order to extrapolate their effect on network efficiency. The table 2 shows the summary of the total power consumption for MRHOF and proposed protocol.
TABLE II

The Total Power Consumption OF THE MRHOF AND PROPOSED PROTOCOL

\begin{tabular}{c|c|c|c}
\hline $\begin{array}{c}\text { Number } \\
\text { of } \\
\text { Nodes }\end{array}$ & $\begin{array}{c}\text { Transmission } \\
\text { Range (m) }\end{array}$ & $\begin{array}{c}\text { MRHOF Total } \\
\text { Power Consumption } \\
(\mathrm{mw})\end{array}$ & $\begin{array}{c}\text { Proposed } \\
\text { Protocol Total } \\
\text { Power Consumption } \\
(\mathrm{mw})\end{array}$ \\
\hline \multirow{3}{*}{50} & 40 & 135.534 & 98.147 \\
& 50 & 116.914 & 92.659 \\
& 60 & 102.165 & 86.191 \\
\hline \multirow{2}{*}{60} & 40 & 253.523 & 152.0 .43 \\
& 50 & 264.969 & 132.809 \\
& 60 & 168.15 & 115.581 \\
\hline \multirow{2}{*}{70} & 40 & 313.605 & 169.602 \\
& 50 & 330.924 & 132.135 \\
\hline
\end{tabular}

TABLE III

THE AVERAGE ETX FOR THE MRHOF AND PROPOSED PROTOCOL

\begin{tabular}{c|c|c|c}
\hline $\begin{array}{c}\text { Number } \\
\text { of } \\
\text { Nodes }\end{array}$ & $\begin{array}{c}\text { Transmission } \\
\text { Range (m) }\end{array}$ & $\begin{array}{c}\text { MRHOF Average } \\
\text { ETX }\end{array}$ & $\begin{array}{c}\text { Proposed Protocol } \\
\text { Average ETX }\end{array}$ \\
\hline \multirow{3}{*}{50} & 40 & 52 & 23 \\
& 50 & 35 & 23 \\
\hline \multirow{2}{*}{60} & 60 & 37 & 26 \\
\hline \multirow{2}{*}{70} & 40 & 78 & 29 \\
& 50 & 54 & 27 \\
& 60 & 32 & 21 \\
\hline
\end{tabular}

Figure 5(a, b, c) shows that the Proposed protocol consume less energy than MRHOF protocol, for various transmission ranges for three network topologies $(50,60,70)$ nodes, the MRHOF protocol is still performing poorly in terms of energy consumption. In the proposed protocol the power consumption decreased when the transmission range increased because the proposed protocol makes load balancing and consumes less energy, due to estimates the best parent among preferred nodes and considering a load metric when selecting the route to the sink node.

\section{Average Expected Transmission Count (ETX)}

Figure $6(\mathrm{a}, \mathrm{b}, \mathrm{c})$ shows the Average ETX for various Transmission ranges, for three network topologies (50, 60, 70 nodes). The average ETX was the lowest in the proposed protocol and increased with MRHOF. A smaller ETX means a lower energy consumption, which indicates a better quality of the routes to the sink node. The table 3 shows the summery of the average ETX for both MRHOF and proposed protocol.

This indicates that the MRHOF protocol performance in large scale networks with transmission range less than $60 \mathrm{~m}$ is so bad, but the proposed protocol keeps the high performance in different transmission ranges (has high PDR, Low Packet loss ratio, Low power consumption, and Low ETX in all topologies).

\section{CONCLUSION}

A brief overview of the RPL routing protocol is presented in this paper and an improvement of the MRHOF in the existing 


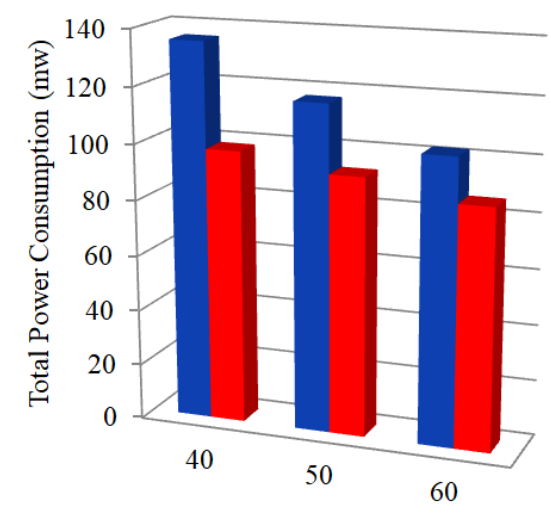

Transmission Range (m)

(a) 50 node

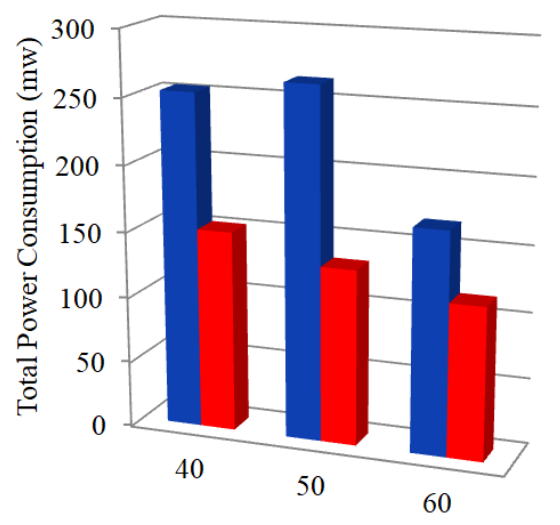

Transmission Range (m)

(b) 60 node

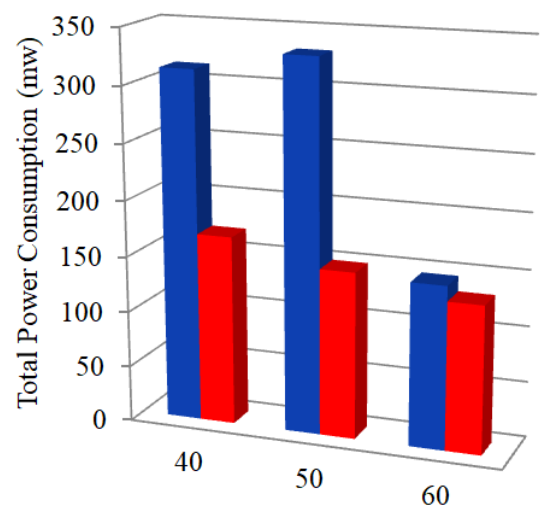

Transmission Range (m)

(c) 70 node

Fig. 5. (a, b, c) The total power consumption for various transmission ranges for three topologies $(50,60,70$ nodes $)$.)

RPL protocol is proposed. In addition, a configuration based on a combination of three metrics (load, residual energy, ETX) is carried out and simulations are carried out in Instant Contiki $3 /$ Cooja, the results indicate that in all network scenarios $(50,60,70$ nodes $)$, with various transmission ranges $(40 \mathrm{~m}, 50 \mathrm{~m}$,
MRHOF

- Proposed Protocol

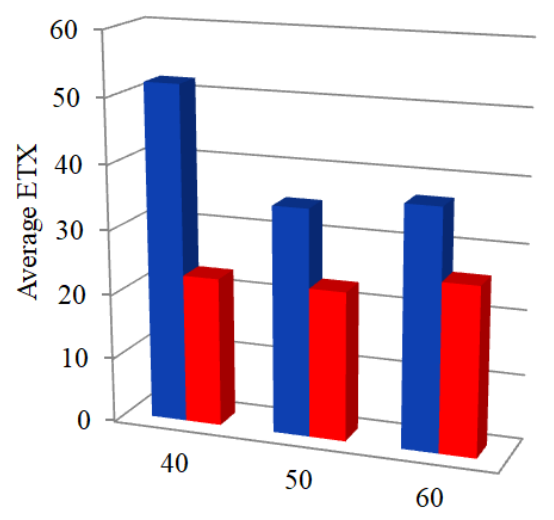

(a) 50 node

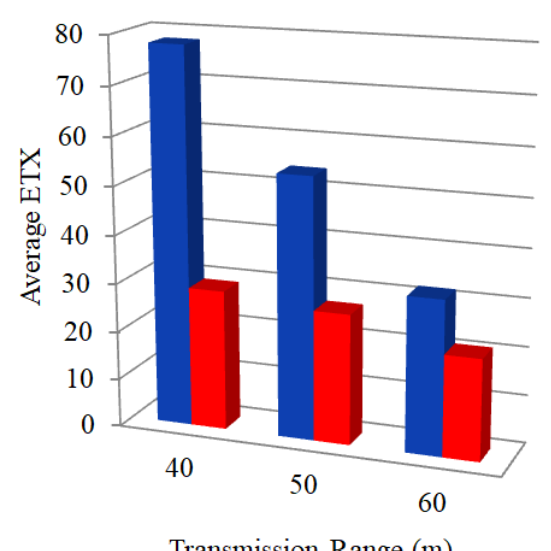

- MRHOF

- Proposed Protocol
MRHOF

- Proposed Protocol
Transmission Range (m)

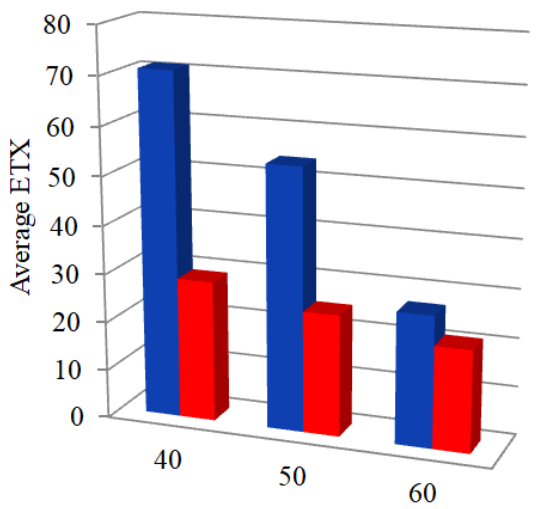

Transmission Range (m)
Transmission Range (m) (b) 60 node

(c) 70 node
- MRHOF

- Proposed Protocol
Fig. 6. $(\mathrm{a}, \mathrm{b}, \mathrm{c})$ The average ETX with various transmission range for three topologies $(50,60,70$ nodes $)$

and $60 \mathrm{~m}$ ), the proposed protocol will effectively select a better routing path and achieve the goal of lower packet loss ratios, higher packet delivery ratios, lower average ETX and lower power consumption than the original RPL protocol. The results show that the efficiency of the proposed protocol increased 
when the number of nodes increased especially with the low transmission ranges $(40,50)$, the PDR increased when the transmission range increased while the power consumption and packet loss ratio decreased because the network hop count to the sink node decreased. In the case of the transmission range is $60 \mathrm{~m}$, most of the nodes reach the sink in one hop, and the rest of the nodes need two hops, so the effect of the proposed protocol is not clearly visible in the case of packet delivery ratio calculation. Other approaches may be used to get high protocol efficiency and conserve more energy, for future work the following points are suggested: Proposing an efficient trickle time algorithm that provides trickle DIO time to increase efficiency more, and it would fascinating to research the RPL behavior with mobility models.

\section{REFERENCES}

[1] Tim Winter, Pascal Thubert, Anders Brandt, Jonathan W Hui,Richard Kelsey, Philip Levis, Kris Pister, Rene Struik, Jean-Philippe Vasseur, Roger K Alexander, et al., "RPL: IPv6 Routing Protocol for Low-Power and Lossy Networks," RFC 6550, pp. 1-157, 2012.

[2] Kris Pister, Pascal Thubert, Sicco Dwars, and Tom Phinney, "Industrial Routing Requirements in Low-Power and Lossy Networks", 2009.

[3] Khadak Singh Bhandari, In-Ho Ra, and Gihwan Cho, "Multitopology Based QoS-Differentiation in RPL for Internet of Things Applications," IEEE Access, vol. 8, pp. 96686-96705, 2020. https://doi.org/10.1109/ACCESS.2020.2995794

[4] Jean-Philippe Vasseur and Adam Dunkels, "Interconnecting Smart Objects with IP: The next internet," Morgan Kaufmann, 2010. https://doi.org/10.1016/C2009-0-20667-2

[5] Pascal Thubert et al., "Objective Function Zero for the Routing Protocol for Low-Power and Lossy Networks (RPL)," 2012

[6] Omprakash Gnawali and Philip Levis, "The Minimum Rank with Hysteresis Objective Function,” RFC 6719, 2012.

[7] Patrick Olivier Kamgueu, Emmanuel Nataf, Thomas Ndie Djotio, and Olivier Festor, "Energy-Based Metric for the Routing Protocol in Low-Power and Lossy Network", Sensornets, pp. 145-148, 2013. https://doi.org/10.5220/0004313401450148

[8] Oana Iova, Fabrice Theoleyre, and Thomas Noel, "Using Multiparent Routing in RPL to Increase the Stability and the Lifetime of the Network," Ad Hoc Networks, vol. 29, pp. 45-62, 2015 https://doi.org/10.1016/j.adhoc.2015.01.020

[9] Fadwa Boubekeur, Lelia Blin, Remy Leone, and Paolo Medagliani, "Bounding Degrees on RPL," Proceedings of the 11th ACM Symposium on QoS and Security forWireless and Mobile Networks, pp. 123-130, 2015. https://doi.org/10.1145/2815317.2815339

[10] Abdullah MA Awad, Rosminazuin Ab Rahim, and Aisha Hassan Abdalla Hashim, "Queue Backlog as A Node Metric for RPL Protocol," International IEEE Conference on Computer and Communication Engineering (ICCCE), pp. 246-250, 2016.https://doi.org/10.1109/ICCCE.2016.61

[11] Mamoun Qasem, Ahmed Al-Dubai, Imed Romdhani, Baraq Ghaleb, and Wajeb Gharibi, "A New Efficient Objective Function for Routing in Internet of Things Paradigm," IEEE conference on standards for communications and networking (CSCN), pp. 1-6, 2016 https://doi.org/10.1109/CSCN.2016.7785168

[12] Hanane Lamaazi and Nabil Benamar, "RPL Enhancement Using A New Objective Function Based on Combined Metrics," 13th IEEE international wireless communications and mobile computing conference (IWCMC), pp. 1459-1464, 2017. https://doi.org/10.1109/IWCMC.2017.7986499

[13] TG Harshavardhana, BS Vineeth, SVR Anand, and Malati Hegde, "Power Control and Cross-Layer Design of RPL Objective Function for Low Power and Lossy Networks," 10th IEEE international conference on communication systems and networks (COMSNETS), pp. 214-219, 2018. https://doi.org/10.1109/COMSNETS.2018.8328200

[14] Parmod Singh and Yaw-Chung Chen, "RPL Enhancement for A Parent Selection Mechanism and an Efficient Objective Function," IEEE Sensors Journal, vol. 19, no. 21, pp. 10054-10066, 2019. https://doi.org/10.1109/JSEN.2019.2927498

[15] P. Sanmartin, A. Rojas, L. Fernandez, K. Avila, D. Jabba, and S. Valle, "Sigma Routing Metric for RPL Protocol," Sensors, vol. 18, no. 4, pp. 1-18, 2018. https://doi.org/10.3390/s18041277

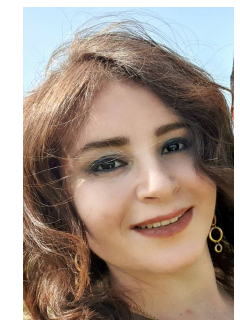

Nariman Najeeb Hadaya received the B.Sc. degree in Computer and Information engineering from Nineveh University, Mosul, Iraq, in 2016. She graduated with high destinations and achieved top one position during her B.Sc. She obtained M.Sc. degree in Computer Engineering from Mosul University in Mosul, Iraq, in 2021. She was an Engineer with Computer and Information Engineering Department, Nineveh University, Mosul, Iraq, from 2016 until September 2019. Currently she is Assistant Lecturer with the eveh University, Mosul, Iraq. Computer and Information engineering from Nin-

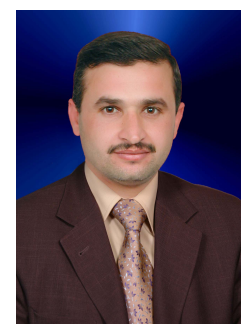

Salah Abdulghani Alabady received the B.Sc. degree in Electronic and Communications Engineering and the M.Sc. degree in Computer Engineering from Mosul University, Mosul, Iraq, in 1996 and 2004 respectively, and the $\mathrm{PhD}$ degree in Wireless Networks from School of Electrical and Electronic Engineering, Universiti Sains Malaysia, Pulau Penang, Malaysia, in May 2014. He was a Lecturer with Computer Engineering Department, Mosul University, Mosul, Iraq, from 1999 until October 2010. Currently, he is Assistant Professor with the Computer Engineering Department, Mosul University, Iraq. His research interests include error correction codes, wireless network coding, joint network - channel coding and cross layer in wireless sensor networks. Dr. Salah has participated in many scientific activities as a reviewer in many respectable publishers such as IEEE, IET, Elsevier, and Springer and many others. Currently, he has more than 55 published papers. He is the supervisor of 7 M.Sc. students and $3 \mathrm{Ph} . \mathrm{D}$. students. 